\title{
Fast Valuation of Forward-Starting Basket Default
}

\author{
Swaps*
}

\author{
Ken Jackson ${ }^{\dagger} \quad$ Alex Kreinin ${ }^{\ddagger} \quad$ Wanhe Zhang ${ }^{\S}$
}

December 13, 2007

\begin{abstract}
A basket default swap (BDS) is a credit derivative with contingent payments that are triggered by a combination of default events of the reference entities. A forwardstarting basket default swap (FBDS) is a BDS starting at a specified future time. Existing analytic or semi-analytic methods for pricing FBDS are time consuming due to the large number of possible default combinations before the BDS starts. This paper develops a fast approximation method for FBDS based on the conditional independence framework. The method converts the pricing of a FBDS to an equivalent BDS pricing problem and combines Monte Carlo simulation with an analytic approach to achieve an effective method. This hybrid method is a novel technique which can be viewed either as a means to accelerate the convergence of Monte Carlo simulation or as a way to estimate parameters in an analytic method that are difficult to compute directly. Numerical results demonstrate the accuracy and efficiency of the proposed hybrid method.
\end{abstract}

\footnotetext{
${ }^{*}$ This research was supported in parted by the Natural Sciences and Engineering Research Council (NSERC) of Canada.

†Department of Computer Science, University of Toronto, 10 King's College Road, Toronto, ON, M5S 3G4, Canada; krj@cs.toronto.edu

¥Algorithmics Inc., 185 Spadina Avenue, Toronto, ON, M5T 2C6, Canada; alex.kreinin@algorithmics.com

$\S$ Department of Computer Science, University of Toronto, 10 King's College Road, Toronto, ON, M5S 3G4, Canada; zhangw@cs.toronto.edu
} 
Keywords: Credit derivatives; forward-starting basket default swaps; conditional independence; hybrid methods.

\section{Introduction}

The credit derivative market has grown explosively during the last 10 years. Among these credit derivatives, the most sophisticated ones are the products associated with a portfolio of underlying assets, such as basket default swaps (BDS) and collateralized debt obligations (CDOs). Recently, new credit derivatives have emerged in more exotic forms, including, for example, forward-starting BDS (FBDS), forward-starting CDOs (FCDOs), options on tranches and leveraged super senior tranches. In this paper, we concentrate on the valuation of FBDS based on the conditional independence framework.

A basket default swap (BDS) is a credit derivative, the underlying assets of which are corporate bonds or other assets subject to credit risk. In an $m$ th-to-default BDS, the protection buyer pays a specified rate (known as the premium or spread) on a specified notional principal periodically until the $m$ th default occurs among the reference entities or until the maturity of the contract. If the $m$ th default happens prior to the maturity of the BDS, the protection seller pays the losses caused by the $m$ th default only to the protection buyer.

A forward-starting BDS is a forward contract obligating the holder to buy or sell a BDS at a specified future time. For example, such a contract might obligate the holder to buy five-year protection on a second-to-default BDS with 10 underlying assets. Suppose that the contract starts one year later and the premium is 100 basis points (bps) per year. During the first year, there is no payment between the buyer and seller. At the end of the first year, if three names in the underlying pool have defaulted, the forward contract obligates the holder to enter a five-year second-to-default BDS on the remaining seven reference entities. The premium is 100 bps per year on the outstanding notional value.

In this paper, we denote the maturity date of the forward contract, or equivalently the starting date of the BDS, by $T$; the maturity date of the BDS by $T^{*}$; and the premium 
dates by $T_{i}, i=1, \ldots, n$, where $T=T_{0}<T_{1}<\ldots<T_{n}=T^{*}$. Figure 1 illustrates possible scenarios for an $m$ th-to-default FBDS. Whether the BDS starts or not is determined by the number of entities left in the basket at $T$ : if less than $m$ names survive till $T$, the contract terminates without any payments as shown in case (a); if at least $m$ entities survive till $T$, the BDS starts, and the cash flows are the same as those for a BDS starting at $T$, as shown in cases (b) and (c).

(a) Less than $m$ entities survive till $T$

$$
0 \quad T_{0}=T
$$

(b) At least $m$ entities survive till $T$ and the $m$ th default does not occur in $\left[T, T^{*}\right]$ Spreads

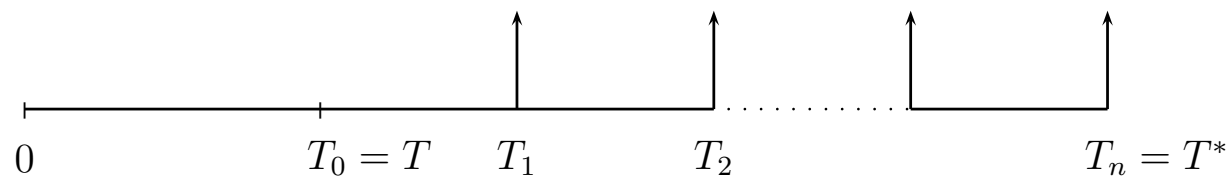

(c) At least $m$ entities survive till $T$ and the $m$ th default occurs in $\left[T, T^{*}\right]$

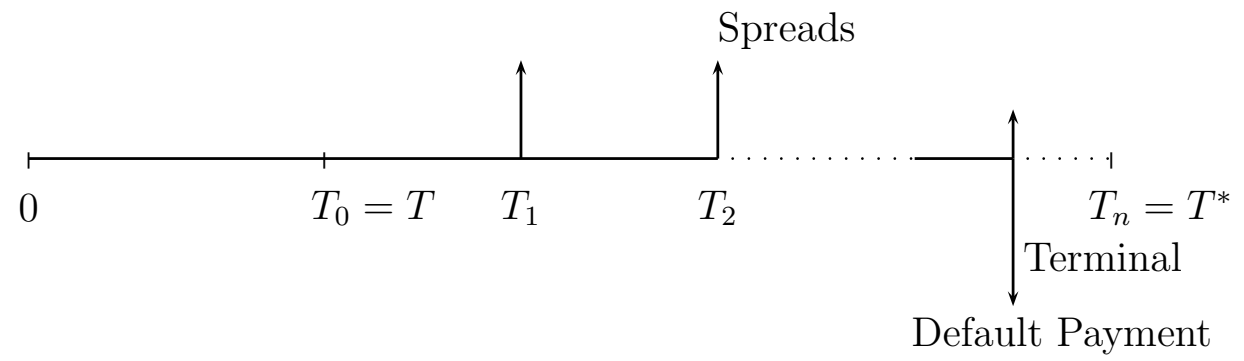

Figure 1: Cash flows for an $m$ th-to-default FBDS

In this paper, if all the underlying names in the reference pool have identical loss-givendefaults, identical default correlations and identical risk-neutral default probabilities, the pool is called completely homogeneous. If the loss-given-defaults are the same, the pool is termed homogeneous. Otherwise, the pool is referred to as inhomogeneous or heterogeneous.

Pricing and hedging of FBDS has become an active research area. The most common approach is Monte Carlo simulation. Such methods are flexible, but are computationally expensive. Therefore, more efficient analytical or semi-analytical approaches are being de- 
veloped by researchers. Zhang [13] developed a "conditional squared method" for FBDS: first conditional on the common factor, then conditional on the outstanding pool at $T$. For a completely homogeneous pool, the method performs effectively. However, for a homogeneous or inhomogeneous pool, the total number of scenarios grows exponentially with the original pool size. Therefore, the method is extremely time consuming: for some $m$, it costs more than a Monte Carlo simulation. A similar conditional squared method was introduced for a reset tranche and a forward-starting tranche by Baheti, Mashal and Naldi [4]. Recently, a more efficient method for pricing a FCDO, that converts it to an equivalent CDO pricing problem, was developed by Andersen [1] and Jackson and Zhang [8], independently. However, due to the contract property, these efficient methods for FCDOs can not be applied to the valuation of FBDS directly.

The object of this paper is to develop a fast approximation method for the valuation of FBDS. The method converts part of the FBDS valuation to an equivalent BDS valuation problem, thereby avoiding the large number of possible default combinations. This transformation approach is a generic method applicable to any model based on the conditional independence framework. To complete the pricing, our new method combines Monte Carlo simulation with the analytic approach to obtain an accurate and efficient hybrid method. The Monte Carlo method generates a coarse approximation for an important parameter in the efficient analytic method. This parameter can not easily be computed directly. Alternatively, the analytic method can be viewed as a means to accelerate the convergence of Monte Carlo simulation. We believe this is the first time that such a hybrid method has been proposed and applied in the quantitative finance area.

The rest of the paper is organized as follows. Section 2 derives the pricing equations for FBDS. Section 3 describes the method to convert a FBDS into an equivalent BDS. Section 4 reviews the widely used Gaussian factor copula model. Section 5 introduces a valuation method for BDS based on the conditional independence framework. Section 6 presents two numerical examples. Section 7 discusses method sensitivity. Section 8 concludes the paper. 


\section{Pricing equations}

We assume the underlying pool contains $K$ instruments with loss-given-default $N_{k}$ for name $k$. Assume that the recovery rates are constant, and the interest rate process is independent of the default process of the basket. Let $d_{i}$ denote the expected value of the risk-free discount factor corresponding to $T_{i}$. Suppose no replacement of the underlying assets in the pool and a constant premium or $\operatorname{spread}^{1} s$. Without loss of generality, we assume that the default payment happens at the nearest premium date following (or equal to) the terminal default time, if it occurs before the contract maturity; and no accrued interest is paid out at the terminal default time.

Let $\tau_{k}$ denote the default time of the $k$ th name and set $\tau_{k}=\infty$, if name $k$ never defaults. The terminal default time $\tau$, which triggers the default payment, can be expressed as a function of individual default times $\tau_{k}$. We denote the loss of the FBDS at the terminal default time by

$$
L= \begin{cases}g\left(N_{k}\right), & \tau=\tau_{k} \in\left(T, T^{*}\right] \\ 0, & \text { otherwise }\end{cases}
$$

where $g(\cdot)$ is a payoff function.

Let $\mathcal{B}_{T}$ denote the set of names left in the basket at $T$. We also denote the number of names in $\mathcal{B}_{T}$ by $\left|\mathcal{B}_{T}\right|$ and the probability distribution of $\mathcal{B}_{T}$ 's composition by $\mathbb{P}\left(\mathcal{B}_{T}\right)$. The event $m \leq\left|\mathcal{B}_{T}\right| \leq K$ is the event that the BDS associated with the FBDS actually starts.

In general, the valuation of a FBDS balances the expectation of the present values of the premium payments (premium leg) against the default payments (default leg), such that $\mathbb{E} V_{\text {prem }}=\mathbb{E} V_{\text {def }}$. Throughout the paper, $\mathbb{E}$ denotes the risk-neutral expectation with respect to the risk-neutral probability $\mathbb{P}$. To compute the expectation numerically, we introduce the terminal default probability

$$
\Pi_{i}^{(k)}=\mathbb{P}\left(\tau=\tau_{k} \in\left(T, T_{i}\right], m \leq\left|\mathcal{B}_{T}\right| \leq K\right)
$$

\footnotetext{
${ }^{1}$ This assumption assists us to compute a fair spread. If we are interested only in computing the value of the contracts, the restriction can be relaxed and a nonconstant spread considered.
} 
We also define the survival indicator function by $\overline{\mathbf{1}}_{i}=\mathbf{1}_{\left\{\tau>T_{i}, m \leq\left|\mathcal{B}_{T}\right| \leq K\right\}}$. Its probability distribution satisfies

$$
\bar{\Pi}_{i}=\mathbb{P}\left(\overline{\mathbf{1}}_{i}=1\right)=\mathbb{P}\left(m \leq\left|\mathcal{B}_{T}\right| \leq K\right)-\sum_{k=1}^{K} \Pi_{i}^{(k)}
$$

Under the assumptions above, the value of the default leg satisfies

$$
\mathbb{E} V_{\text {def }}=\sum_{k=1}^{K} g\left(N_{k}\right) \sum_{i=1}^{n} d_{i}\left(\Pi_{i}^{(k)}-\Pi_{i-1}^{(k)}\right)=\sum_{i=1}^{n} d_{i} \sum_{k=1}^{K} g\left(N_{k}\right)\left(\Pi_{i}^{(k)}-\Pi_{i-1}^{(k)}\right)
$$

Similarly, the value of the premium leg satisfies

$$
\mathbb{E} V_{\text {prem }}=\mathbb{E}\left[s N_{T} \overline{\mathbf{1}}_{i} \sum_{j=1}^{i} \Delta T_{j} \cdot d_{j}\right]=s \sum_{i=1}^{n} \Delta T_{i} \cdot d_{i} \cdot \mathbb{E}\left[N_{T} \overline{\mathbf{1}}_{i}\right]
$$

where $N_{T}$ is the sum of the notational values of all names in $\mathcal{B}_{T}$, and $\Delta T_{i}=T_{i}-T_{i-1}$. Therefore, the fair spread can be computed by

$$
s=\frac{\mathbb{E} V_{\mathrm{def}}}{\mathrm{DV} 01}=\frac{\sum_{i=1}^{n} d_{i} \sum_{k=1}^{K} g\left(N_{k}\right)\left(\Pi_{i}^{(k)}-\Pi_{i-1}^{(k)}\right)}{\sum_{i=1}^{n} \Delta T_{i} \cdot d_{i} \cdot \mathbb{E}\left[N_{T} \overline{\mathbf{1}}_{i}\right]}
$$

where DV01 $\equiv \sum_{i=1}^{n} \Delta T_{i} \cdot d_{i} \cdot \mathbb{E}\left[N_{T} \overline{\mathbf{1}}_{i}\right]$. Alternately, if the spread is set, the value of the FBDS is the difference between the two legs:

$$
V_{\mathrm{fwd}}=\mathbb{E} V_{\text {prem }}-\mathbb{E} V_{\text {def }}
$$

\section{$3 \quad$ FBDS to BDS}

Define $\hat{\Pi}_{i}^{(k)}=\mathbb{P}\left(\tau=\tau_{k} \in\left(T, T_{i}\right]\right)$. Then

$$
\begin{aligned}
\hat{\Pi}_{i}^{(k)} & =\mathbb{P}\left(\tau=\tau_{k} \in\left(T, T_{i}\right], m \leq\left|\mathcal{B}_{T}\right| \leq K\right)+\mathbb{P}\left(\tau=\tau_{k} \in\left(T, T_{i}\right],\left|\mathcal{B}_{T}\right|<m\right) \\
& =\Pi_{i}^{(k)}+\mathbb{P}\left(\tau=\tau_{k} \in\left(T, T_{i}\right],\left|\mathcal{B}_{T}\right|<m\right)
\end{aligned}
$$


However, if $\left|\mathcal{B}_{T}\right|<m$, the $m$ th default could never happen. Therefore,

$$
\mathbb{P}\left(\tau=\tau_{k} \in\left(T, T_{i}\right],\left|\mathcal{B}_{T}\right|<m\right)=0
$$

Hence, we obtain $\Pi_{i}^{(k)}=\hat{\Pi}_{i}^{(k)}$. Therefore, we can compute $\Pi_{i}^{(k)}$ through $\hat{\Pi}_{i}^{(k)}$, which is independent of $\mathcal{B}_{T}$. Most importantly, $\hat{\Pi}_{i}^{(k)}$ is defined in the same form as a similar probability used to value BDS in [6] and [9] with $T=0$. Therefore, we can use the method for BDS to compute the key probability $\Pi_{i}^{(k)}$ with modified default probabilities. That is, instead of using the probability of name $k$ defaulting before time $t$, we use the probability of name $k$ defaulting in $(T, t]$. The starting pool of the BDS associated with the FBDS is random in the original problem; after the transformation, the starting pool in the equivalent BDS contains all the $K$ names, which becomes certain. Therefore, we avoid the large combinatorial problem due to the consideration of all the possible starting pools in the original formulation of the problem.

To illustrate the transformation, we compute $\Pi_{i}^{(k)}$ for a simple first-to-default FBDS and its equivalent BDS. We assume $\tau_{k}$ are independent of each other and follow an exponential distribution with constant intensity $\lambda_{k}$ over time. Then, $\mathbb{P}\left(\tau_{k} \in(t, t+d t]\right)=\lambda_{k} \exp \left(-\lambda_{k} t\right) d t$ for an infinitesimal $d t$. Without the transformation, the event $\tau=\tau_{k} \in(t, t+d t]$ for the first-to-default FBDS is equivalent to the event $\left(\tau_{k} \in(t, t+d t]\right) \wedge\left(\left(\tau_{j} \in(0, T]\right) \vee\left(\tau_{j}>\right.\right.$ $t+d t), \forall j \neq k)$. Therefore,

$$
\begin{aligned}
\mathbb{P}\left(\tau=\tau_{k} \in(t, t+d t]\right) & =\mathbb{P}\left(\tau_{k} \in(t, t+d t]\right) \prod_{j \neq k}\left(\mathbb{P}\left(\tau_{j} \in(0, T]\right)+\mathbb{P}\left(\tau_{j}>t+d t\right)\right) \\
& =\lambda_{k} \exp \left(-\lambda_{k} t\right) d t \prod_{j \neq k}\left(1-\exp \left(-\lambda_{j} T\right)+\exp \left(-\lambda_{j}(t+d t)\right)\right)
\end{aligned}
$$

With the transformation, the modified probability $\hat{\pi}_{k}(t) \equiv \mathbb{P}\left(\tau_{k} \leq t\right)-\mathbb{P}\left(\tau_{k} \leq T\right)$ satisfies

$$
\hat{\pi}_{k}(t)=\exp \left(-\lambda_{k} T\right)-\exp \left(-\lambda_{k} t\right)
$$


The default intensity for $\hat{\pi}_{k}(t)$ is still $\lambda_{k}$. However, these $\hat{\pi}_{k}(t)$ are for the equivalent BDS. In the BDS, the event $\tau=\tau_{k} \in(t, t+d t]$ is equivalent to the event $\left(\tau_{k} \in(t, t+d t]\right) \wedge\left(\tau_{j}>\right.$ $t+d t, \forall j \neq k)$. Therefore,

$$
\begin{aligned}
\mathbb{P}\left(\tau=\tau_{k} \in(t, t+d t]\right) & =\mathbb{P}\left(\tau_{k} \in(t, t+d t]\right) \prod_{j \neq k}\left(\mathbb{P}\left(\tau_{j}>t+d t\right)\right) \\
& =\lambda_{k} \exp \left(-\lambda_{k} t\right) d t \prod_{j \neq k}\left(1-\hat{\pi}_{j}(t+d t)\right)
\end{aligned}
$$

which is equal to (5). Therefore, $\Pi_{i}^{(k)}=\int_{T}^{T_{i}} \mathbb{P}\left(\tau=\tau_{k} \in(t, t+d t]\right)$ in the original FBDS is the same as that in the equivalent BDS, which supports the correctness of the transformation.

Once $\Pi_{i}^{(k)}$ is known, the computation of $\mathbb{E} V_{\text {def }}$ is straightforward following (2). To compute $\mathbb{E} V_{\text {prem }}$ or DV01, we need to compute the expectation $\mathbb{E}\left[N_{T} \overline{\mathbf{1}}_{i}\right]$. Suppose we know the correlation $\rho_{i}$ between $N_{T}$ and $\overline{\mathbf{1}}_{i}$, then $\mathbb{E}\left[N_{T} \overline{\mathbf{1}}_{i}\right]$ can be computed from

$$
\rho_{i}=\frac{\mathbb{E}\left[N_{T} \overline{\mathbf{1}}_{i}\right]-\mathbb{E}\left[N_{T}\right] \mathbb{E}\left[\overline{\mathbf{1}}_{i}\right]}{\sqrt{\operatorname{var}\left(N_{T}\right) \operatorname{var}\left(\overline{\mathbf{1}}_{i}\right)}}
$$

where $\mathbb{E}\left[\overline{\mathbf{1}}_{i}\right]=\bar{\Pi}_{i}$ and $\operatorname{var}\left(\overline{\mathbf{1}}_{i}\right)=\bar{\Pi}_{i}\left(1-\bar{\Pi}_{i}\right)$. Once $\Pi_{i}^{(k)}$ is known, the computation of $\bar{\Pi}_{i}$ defined in (1) is straightforward, since the term $\mathbb{P}\left(m \leq\left|\mathcal{B}_{T}\right| \leq K\right)$ can be computed by the pool loss distribution methods for CDOs, e.g., [3], [10], [5] and [7]. Similarly, the terms $\mathbb{E}\left[N_{T}\right]$ and $\operatorname{var}\left(N_{T}\right)$ are easy to compute with known pool loss distribution. Therefore, $\mathbb{E}\left[N_{T} \overline{\mathbf{1}}_{i}\right]$ can be computed by

$$
\mathbb{E}\left[N_{T} \overline{\mathbf{1}}_{i}\right]=\mathbb{E}\left[N_{T}\right] \bar{\Pi}_{i}+\rho_{i} \sqrt{\operatorname{var}\left(N_{T}\right) \bar{\Pi}_{i}\left(1-\bar{\Pi}_{i}\right)}
$$

Hence, we can compute the premium leg value and complete the valuation.

The only unknown variable in $(7)$ is the correlation coefficient $\rho_{i}$. We propose to use Monte Carlo simulation to approximate $\rho_{i}$. As we show later, the spread is not sensitive to the value of the correlation coefficients. Therefore, a rough approximation only to the $\rho_{i}$ is needed. This is an important property of this application which contributes to the 
effectiveness of our hybrid method.

To compute the value of $\hat{\Pi}_{i}^{(k)}$ for FBDS, we need to compute the joint distribution of $K$ correlated random variables $\mathbf{1}_{\left\{T<\tau_{k} \leq T_{i}\right\}}$. One effective approach is the conditional independence framework. In the next section, we review the market-standard Gaussian factor copula model — one example of the conditional independence framework.

\section{Gaussian factor copula model}

Due to their tractability, Gaussian factor copula models are widely used to specify a joint distribution for default times consistent with their marginal distribution. A one factor model was first introduced by Vasicek [12] to evaluate the loan loss distribution, and the Gaussian copula was first applied to multi-name credit derivatives by $\mathrm{Li}$ [11]. After that, the model was generalized by Andersen, Sidenius and Basu [3], Andersen and Sidenius [2], Hull and White [5], and Laurent and Gregory [10], to name just a few.

Assume the risk-neutral (cumulative) default probabilities

$$
\pi_{k}(t)=\mathbb{P}\left(\tau_{k} \leq t\right), \quad k=1,2, \ldots, K
$$

are known. To generate the dependence structure of default times, we introduce random variables $U_{k}$ satisfying

$$
U_{k}=\beta_{k} X+\sigma_{k} \varepsilon_{k}, \quad \text { for } k=1,2, \ldots, K
$$

where $X$ is the systematic risk factor reflecting the health of the macroeconomic environment; $\varepsilon_{k}$ are idiosyncratic risk factors, which are independent of each other and also independent

of $X$; the constants $\beta_{k}$ and $\sigma_{k}$, satisfying $\beta_{k}^{2}+\sigma_{k}^{2}=1$, are assumed to be known. The random variables $X$ and $\varepsilon_{k}$ are assumed to follow zero-mean unit-variance distributions, so the correlation between $U_{i}$ and $U_{j}$ is $\beta_{i} \beta_{j}$.

The default times $\tau_{k}$ and the random variables $U_{k}$ are connected by a percentile-to- 
percentile transformation, such that

$$
\pi_{k}(t)=\mathbb{P}\left(\tau_{k} \leq t\right)=\mathbb{P}\left(U_{k} \leq u_{k}(t)\right)
$$

where each $u_{k}(t)$ can be viewed as a default barrier. Thus the dependence among default times is captured by the common factor $X$. In the Gaussian factor copula model, we assume $X$ and $\varepsilon_{k}$ follow standard normal distributions. Consequently, each $U_{k}$ also follows a standard normal distribution. Hence we have

$$
u_{k}(t)=\Phi^{-1}\left(\pi_{k}(t)\right) .
$$

where $\Phi$ is the standard normal cumulative distribution function.

Conditional on a particular value $x$ of $X$, the conditional risk-neutral default probabilities are defined as

$$
\pi_{k}(t, x) \equiv \mathbb{P}\left(\tau_{k} \leq t \mid X=x\right)=\mathbb{P}\left(U_{k} \leq u_{k}(t) \mid X=x\right)
$$

Substituting (8) and (9) into (10), we have

$$
\pi_{k}(t, x)=\mathbb{P}\left[\beta_{k} x+\sigma_{k} \varepsilon_{k} \leq \Phi^{-1}\left(\pi_{k}(t)\right)\right]=\Phi\left[\frac{\Phi^{-1}\left(\pi_{k}(t)\right)-\beta_{k} x}{\sigma_{k}}\right]
$$

This is an example of a conditional independence framework: the default events of the names are assumed to be conditionally independent. Thus, the problem of correlated names is reduced to the problem of independent names. By (2) and (3), the mean values of the default leg and premium leg for a FBDS can be evaluated as

$$
\mathbb{E} V_{\mathrm{def}}=\int_{-\infty}^{\infty} \mathbb{E}_{x}\left[V_{\mathrm{def}}\right] d \Phi(x)=\int_{-\infty}^{\infty} \sum_{i=1}^{n} d_{i}\left(\sum_{k=1}^{K} g\left(N_{k}\right)\left(\Pi_{i}^{(k)}(x)-\Pi_{i-1}^{(k)}(x)\right)\right) d \Phi(x)
$$




$$
\begin{aligned}
\mathbb{E} V_{\text {prem }} & =s \sum_{i=1}^{n} \Delta T_{i} \cdot d_{i}\left(\mathbb{E}\left[N_{T}\right] \bar{\Pi}_{i}+\rho_{i} \sqrt{\operatorname{var}\left(N_{T}\right) \bar{\Pi}_{i}\left(1-\bar{\Pi}_{i}\right)}\right) \\
& =s \sum_{i=1}^{n} \Delta T_{i} \cdot d_{i}\left(\int_{-\infty}^{\infty} \mathbb{E}_{x}\left[N_{T}\right] \bar{\Pi}_{i}(x) d \Phi(x)+\rho_{i} \int_{-\infty}^{\infty} \sqrt{\operatorname{var}_{x}\left(N_{T}\right) \bar{\Pi}_{i}(x)\left(1-\bar{\Pi}_{i}(x)\right)} d \Phi(x)\right) \\
& =\int_{-\infty}^{\infty} s \sum_{i=1}^{n} \Delta T_{i} \cdot d_{i}\left(\mathbb{E}_{x}\left[N_{T}\right] \bar{\Pi}_{i}(x)+\rho_{i} \sqrt{\operatorname{var}_{x}\left(N_{T}\right) \bar{\Pi}_{i}(x)\left(1-\bar{\Pi}_{i}(x)\right)}\right) d \Phi(x) \\
& =\int_{-\infty}^{\infty} \mathbb{E}_{x}\left[V_{\text {prem }}\right] d \Phi(x)
\end{aligned}
$$

where $\mathbb{E}_{x}$ denotes the risk-neutral expectation with respect to the risk-neutral probability $\mathbb{P}_{x}$, conditional on $X=x$. For simplicity, we denote the integrand of (13) by $\mathbb{E}_{x}\left[V_{\text {prem }}\right]$. However, it is essential for computational efficiency that we use the unconditional $\rho_{i}$ in (13), rather than the conditional $\rho_{i}(x)$, as would be expected from the definition of $\mathbb{E}_{x}\left[V_{\text {prem }}\right]$.

To approximate the integrals (12) and (13), we use a quadrature rule, such as the Gaussian-Legendre rule or the Gaussian-Hermite rule. Thus, for example, the integral (12) reduces to

$$
\mathbb{E} V_{\text {def }} \approx \sum_{m=1}^{M} w_{m} \mathbb{E}_{x_{m}}\left[V_{\text {def }}\right]
$$

where the $w_{m}$ and $x_{m}$ are the quadrature weights and nodes, respectively. Therefore, the main challenge in pricing a FBDS lies in computing $\mathbb{P}_{x}\left(\left|\mathcal{B}_{T}\right|\right), \mathbb{P}_{x}\left(N_{T}\right), \Pi_{i}^{(k)}(x)$ and $\bar{\Pi}_{i}(x)$, conditional on a given value $x$ of $X$.

\subsection{Conditional forward default probabilities}

Conditional on a given $x$, to compute the distribution of $\hat{\Pi}_{i}^{(k)}$, we need to specify the distribution of $\mathbf{1}_{\left\{T<\tau_{k} \leq T_{i}\right\}}$, which is equal to the conditional distribution of $\mathbf{1}_{\left\{u_{k}(T)<U_{k} \leq u_{k}\left(T_{i}\right)\right\}}$. To this end, we introduce conditional forward default probabilities

$$
\hat{\pi}_{k}(t, x)=\pi_{k}(t, x)-\pi_{k}(T, x), \quad \text { for } t \geq T
$$

so that the conditional distribution of $\mathbf{1}_{\left\{T<\tau_{k} \leq T_{i}\right\}}$ satisfies $\mathbb{P}_{x}\left(\mathbf{1}_{\left\{T<\tau_{k} \leq T_{i}\right\}}=1\right)=\hat{\pi}_{k}\left(T_{i}, x\right)$. Armed with the conditional forward default probabilities, the conditional terminal default 
probability $\hat{\Pi}_{i}^{(k)}$ for a FBDS can be computed using the methods developed for BDS discussed in the next section. With the conditional default probabilities $\pi_{k}(T, x)$, the conditional distribution of $N_{T}$ and $\left|\mathcal{B}_{T}\right|$ can also be computed using the methods for CDOs or by brute force to explore all the possible combinations.

\subsection{Conditional forward default intensities}

Besides the conditional forward default probabilities, to compute the conditional probability $\hat{\Pi}_{i}^{(k)}(x)$ by the methods for BDS, we need to introduce the conditional forward default intensities. Assume the conditional forward default distribution that name $k$ defaults in $(T, t]$ follows the Cox process

$$
\mathbb{P}_{x}\left(T<\tau_{k} \leq t\right)=1-\exp \left(-\Lambda_{k}(t, x)\right)
$$

where

$$
\Lambda_{k}(t, x)=\int_{T}^{t} \lambda_{k}(u, x) d u
$$

and $\lambda_{k}(\cdot)$ is the conditional forward default intensity of the $k$ th name. We know

$$
\mathbb{P}_{x}\left(T<\tau_{k} \leq t\right)=\hat{\pi}_{k}(t, x)
$$

where $\hat{\pi}_{k}(t, x)$ is given by $(15)$. If we assume $\Lambda_{k}(t, x)$ is linear between premium dates $T_{i}$, then (17) implies that $\lambda_{k}(t, x)$ is a piecewise constant function, such that

$$
\lambda_{k}(t, x)=\lambda_{k}\left(T_{i}, x\right), \quad \text { for } t \in\left(T_{i-1}, T_{i}\right]
$$

Combining this result with (17), we have

$$
\Lambda_{k}\left(T_{i}, x\right)=\Lambda_{k}\left(T_{i-1}, x\right)+\lambda_{k}\left(T_{i}, x\right) \cdot \Delta T_{i}
$$


from which, we obtain

$$
\lambda_{k}\left(T_{i}, x\right)=\frac{1}{\Delta T_{i}}\left(\Lambda_{k}\left(T_{i}, x\right)-\Lambda_{k}\left(T_{i-1}, x\right)\right)
$$

From (16) and (18), we know

$$
\begin{gathered}
\Lambda_{k}\left(T_{i-1}, x\right)=-\ln \left(1-\hat{\pi}_{k}\left(T_{i-1}, x\right)\right) \\
\Lambda_{k}\left(T_{i}, x\right)=-\ln \left(1-\hat{\pi}_{k}\left(T_{i}, x\right)\right)
\end{gathered}
$$

Substituting these expressions for $\Lambda_{k}\left(T_{i-1}, x\right)$ and $\Lambda_{k}\left(T_{i}, x\right)$ into (19), we obtain

$$
\lambda_{k}\left(T_{i}, x\right)=\frac{1}{\Delta T_{i}} \ln \left(\frac{1-\hat{\pi}_{k}\left(T_{i-1}, x\right)}{1-\hat{\pi}_{k}\left(T_{i}, x\right)}\right), \quad \text { for } i=1,2, \ldots, n
$$

\section{Terminal default probabilities}

Available methods for BDS include the convolution technique by Laurent and Gregory [10] and the recursive method based on the order statistics of individual default times by Iscoe and Kreinin [6]. Here we review the recursive method in [6] and use it in our numerical examples.

In a first-to-default BDS, the conditional probabilities $\Delta \hat{\Pi}_{i}^{(k)}(x)=\mathbb{P}_{x}\left(\tau=\tau_{k} \in\left(T_{i-1}, T_{i}\right]\right)$ satisfy

$$
\Delta \hat{\Pi}_{i}^{(k)}(x)=\hat{\Pi}_{i}^{(k)}(x)-\hat{\Pi}_{i-1}^{(k)}(x)=\frac{\lambda_{k}\left(T_{i}, x\right)}{\sum_{k=1}^{K} \lambda_{k}\left(T_{i}, x\right)}\left(\bar{\Pi}_{i-1}(x)-\bar{\Pi}_{i}(x)\right)
$$

where $\lambda_{k}(\cdot)$ is the conditional forward default intensities defined in $(20)$; and $\bar{\Pi}_{i}(x)=$ $\prod_{k=1}^{K}\left(1-\hat{\pi}_{k}\left(T_{i}, x\right)\right)$.

For the $m$ th-to-default BDS, Iscoe and Kreinin [6] derive the recursive relation between the $m$ th-to-default and the $(m-1)$ st-to-default contracts:

$$
(m-1) \mathcal{P}_{m}(\mathcal{B})=\sum_{j \neq k} \mathcal{P}_{m-1}\left(\mathcal{B}^{[j]}\right)-(K-m+1) \mathcal{P}_{m-1}(\mathcal{B})
$$


where $\mathcal{P}_{m}(\mathcal{B})=\mathbb{P}\left(\tau=\tau_{k} \in\left(T_{i-1}, T_{i}\right]\right)$ for the $m$ th-to-default BDS; and $\mathcal{B}^{[j]}$ is the set of names obtained by excluding name $j$ from $\mathcal{B}$. Naive implementation of this recursion causes the recalculation of the same probabilities. To avoid the recalculation, use

$$
\mathcal{P}_{m}(\mathcal{B})=\sum_{v=0}^{m-1}(-1)^{m-v-1}\left(\begin{array}{l}
K-v-1 \\
m-v-1
\end{array}\right) \sum_{\mathcal{J} \subset \mathcal{B}:|\mathcal{J}|=v} \mathcal{P}_{1}\left(\mathcal{B}^{[\mathcal{J}]}\right)
$$

where $\mathcal{J}$ is a subset of $\mathcal{B}$ and $\mathcal{B}^{[\mathcal{J}]}=\mathcal{B} \backslash \mathcal{J}$. Here, for simplicity, we give the recursion for the unconditional probabilities, but a similar recursion is also valid for the conditional probabilities.

\section{$6 \quad$ Numerical results}

Based on the methods described above, we propose the following steps for pricing FBDS:

1. Convert $\pi_{k}\left(T_{i}\right)$ to conditional default probabilities $\pi_{k}\left(T_{i}, x\right)$ using (11) and then compute the conditional forward default probabilities $\hat{\pi}_{k}\left(T_{i}, x\right)$ using (15);

2. Compute the conditional distribution $\Pi_{i}^{(k)}(x)$ by the recursive method in Section 5 and $\mathbb{P}_{x}\left(m \leq\left|\mathcal{B}_{T}\right| \leq K\right)$ and $\mathbb{P}_{x}\left(N_{T}\right)$ by the methods for CDOs as well as $\bar{\Pi}_{i}(x)$ using (1), $\mathbb{E}_{x}\left[N_{T}\right]$ and $\operatorname{var}_{x}\left(N_{T}\right)$ from $\mathbb{P}_{x}\left(N_{T}\right) ;$

3. Run a Monte Carlo simulation to approximate the $\rho_{i}$ in (6);

4. Evaluate $\mathbb{E}_{x}\left[V_{\text {def }}\right]$ and $\mathbb{E}_{x}\left[V_{\text {prem }}\right]$ by (12) and (13), respectively;

5. Approximate $\mathbb{E} V_{\text {def }}$ and $\mathbb{E} V_{\text {prem }}$ using a quadrature rule (see (14));

6. Complete the computation using the pricing equations (4).

The numerical experiments are based on two FBDS: one is a homogeneous pool; the other is an inhomogeneous pool. The contracts are 5-year BDS starting one year later with quarterly premium payments, i.e., $T=T_{0}=1, T_{i}=1+0.25 i$, for $i=1, \ldots, 20$. The continuously compounded interest rates are $4 \%$ for each $T_{i}$. The recovery rate of the 


\begin{tabular}{|r|ccc|}
\hline Name & Notional & Credit Rating & $\beta_{k}$ \\
\hline 1 & 190 & $\mathrm{C} 4$ & 0.5 \\
2 & 80 & $\mathrm{C} 6$ & 0.6 \\
3 & 70 & $\mathrm{C} 1$ & 0.9 \\
4 & 360 & $\mathrm{C} 5$ & 0.6 \\
5 & 100 & $\mathrm{C} 2$ & 0.5 \\
6 & 200 & $\mathrm{C} 5$ & 0.4 \\
7 & 150 & $\mathrm{C} 5$ & 0.7 \\
8 & 123 & $\mathrm{C} 2$ & 0.64 \\
9 & 95 & $\mathrm{C} 5$ & 0.55 \\
10 & 107 & $\mathrm{C} 8$ & 0.22 \\
\hline
\end{tabular}

Table 1: Inhomogeneous FBDS pool

\begin{tabular}{|c|cccccc|}
\hline & \multicolumn{6}{|c|}{ Time } \\
\cline { 2 - 7 } Rating & 1 & 2 & 3 & 4 & 5 & 6 \\
\hline C1 & 0.004535 & 0.011203 & 0.018947 & 0.027044 & 0.035605 & 0.045778 \\
C2 & 0.005102 & 0.012604 & 0.021315 & 0.030425 & 0.040056 & 0.053843 \\
C3 & 0.006802 & 0.016805 & 0.028420 & 0.040567 & 0.053408 & 0.070828 \\
C4 & 0.009063 & 0.022369 & 0.037786 & 0.053868 & 0.070828 & 0.088059 \\
C5 & 0.009704 & 0.023926 & 0.040369 & 0.057481 & 0.075479 & 0.100592 \\
C6 & 0.010876 & 0.026843 & 0.045343 & 0.064642 & 0.084993 & 0.105671 \\
C7 & 0.013586 & 0.033497 & 0.056517 & 0.080473 & 0.105671 & 0.132089 \\
C8 & 0.013595 & 0.033553 & 0.056679 & 0.080802 & 0.106241 & 0.144459 \\
\hline
\end{tabular}

Table 2: Part of risk-neutral cumulative default probabilities

instruments in the pool is $15 \%$. The pool structure of the inhomogeneous FBDS is defined in Table 1; the homogeneous pool has the same structure except that the notional values are 100 for all names. Part of the risk-neutral cumulative default probabilities for different credit ratings are listed in Table 2. The risk-neutral default probabilities for all time points can be found at www.cs.toronto.edu/NA/DefaultProb.html.

Table 3 lists the premiums for the $m$ th-to-default FBDS $(m=1, \ldots, 4)$ computed by the conditional squared method of Zhang [13] (column "Analytic"), and our fast approximation method described above (column "Approximation") with $10^{3}$ trials in the Monte Carlo simulation to approximate the correlation coefficients $\rho_{i}$. The table also lists the $95 \%$ confidence interval of the spread computed by a Monte Carlo method (column "95\% CI"). The $95 \%$ confidence interval is computed as follows: each Monte Carlo experiment consists of $10^{6}$ trials; we repeat each Monte Carlo experiment 500 times; then, we compute the $95 \%$ 


\begin{tabular}{|l|ccrrc|}
\hline Pool & $m$ & $95 \%$ CI & Analytic & Approximation & Rel Err \\
\hline \multirow{3}{*}{ Homogeneous } & 1 & {$[104.66,105.35]$} & 105.00 & 105.07 & $6.98 \times 10^{-4}$ \\
Pool & 2 & {$[35.70,36.08]$} & 35.90 & 35.92 & $7.00 \times 10^{-4}$ \\
& 3 & {$[14.80,15.08]$} & 14.94 & 14.94 & $3.16 \times 10^{-4}$ \\
& 4 & {$[6.29,6.46]$} & 6.38 & 6.38 & $2.81 \times 10^{-5}$ \\
\hline \multirow{3}{*}{ Inhomogeneous } & 1 & {$[108.79,109.75]$} & 109.27 & 109.24 & $2.50 \times 10^{-4}$ \\
Pool & 2 & {$[37.23,37.72]$} & 37.45 & 37.45 & $1.38 \times 10^{-4}$ \\
& 3 & {$[15.18,15.46]$} & 15.32 & 15.32 & $7.52 \times 10^{-5}$ \\
& 4 & {$[6.35,6.57]$} & 6.46 & 6.47 & $3.65 \times 10^{-4}$ \\
\hline
\end{tabular}

Table 3: FBDS premiums (bps)

\begin{tabular}{|l|ccccc|}
\hline Pool & Method & $m=1$ & $m=2$ & $m=3$ & $m=4$ \\
\hline \multirow{3}{*}{ Homogeneous } & $95 \%$ CI & {$[104.66,105.35]$} & {$[35.70,36.08]$} & {$[14.80,15.08]$} & {$[6.29,6.46]$} \\
Pool & 100 & {$[104.64,105.42]$} & {$[35.82,35.99]$} & {$[14.92,14.96]$} & {$[6.37,6.38]$} \\
& 1,000 & {$[104.88,105.11]$} & {$[35.87,35.93]$} & {$[14.93,14.95]$} & {$[6.38,6.38]$} \\
& 10,000 & {$[104.96,105.03]$} & {$[35.89,35.91]$} & {$[14.94,14.94]$} & {$[6.38,6.38]$} \\
\hline \multirow{3}{*}{ Inhomogeneous } & $95 \%$ CI & {$[108.79,109.75]$} & {$[37.23,37.72]$} & {$[15.18,15.46]$} & {$[6.35,6.57]$} \\
Pool & 100 & {$[108.89,109.69]$} & {$[37.35,37.55]$} & {$[15.29,15.34]$} & {$[6.46,6.47]$} \\
& 1,000 & {$[109.14,109.42]$} & {$[37.42,37.49]$} & {$[15.31,15.33]$} & {$[6.46,6.47]$} \\
& 10,000 & {$[109.23,109.31]$} & {$[37.44,37.47]$} & {$[15.32,15.33]$} & {$[6.46,6.47]$} \\
\hline
\end{tabular}

Table 4: 95\% confidence interval comparison (bps)

confidence interval from the empirical distribution of those 500 samples. The last column of Table 3 lists the relative errors of the spreads computed by our fast approximation method, using the spreads computed by the analytic method for the exact solution. Table 4 compares the $95 \%$ confidence interval computed by $10^{6}$ trials of Monte Carlo simulation (row " $95 \%$ CI") with those computed by our fast approximation method with 100, 1,000 and 10,000 trials (rows "100", "1,000" and "10,000", respectively). These tables demonstrate that our fast approximation method is accurate for the valuation of FBDS.

For the homogeneous FBDS, the running time of the Monte Carlo simulation with $10^{6}$ trials is about 400 times slower than our fast approximation method; for the inhomogeneous FBDS, the running time of the Monte Carlo simulation is about 20 times slower than our fast approximation method. Our fast approximation method is also faster than the analytic method. For example, for the first-to-default homogeneous FBDS, the running time of the analytic method is about 40 times slower than our fast approximation method. These 
comparisons demonstrate that our fast approximation method for FBDS outperforms both the Monte Carlo method and the analytic method.

\section{Sensitivity analysis}

Since we use a Monte Carlo method to approximate the correlation coefficients $\rho_{i}$, the $\rho_{i}$ are usually not exact. Therefore, a natural question to ask is: how sensitive is the FBDS spread to small changes in the $\rho_{i}$ ? If the sensitivity is weak, then our approximation method can obtain accurate results with a modest amount of work. Weak sensitivity is a key requirement to ensure that this kind of hybrid method is an effective computational approach.

The sensitivity of the spread with respect to small changes in the $\rho_{i}$ is determined by

$$
\begin{aligned}
\frac{\partial s}{\partial \rho_{i}} & =\frac{\partial s}{\partial \mathrm{DV} 01} \frac{\partial \mathrm{DV} 01}{\partial \rho_{i}} \\
& =\frac{\partial\left(\mathbb{E}\left[V_{\mathrm{def}}\right] / \mathrm{DV} 01\right)}{\partial \mathrm{DV} 01} \Delta T_{i} \cdot d_{i} \sqrt{\operatorname{var}\left(N_{T}\right) \bar{\Pi}_{i}\left(1-\bar{\Pi}_{i}\right)} \\
& =-\frac{\mathbb{E}\left[V_{\mathrm{def}}\right]}{\mathrm{DV} 01^{2}} \Delta T_{i} \cdot d_{i} \sqrt{\operatorname{var}\left(N_{T}\right) \bar{\Pi}_{i}\left(1-\bar{\Pi}_{i}\right)} \\
& =-s \frac{\Delta T_{i} \cdot d_{i} \sqrt{\operatorname{var}\left(N_{T}\right) \bar{\Pi}_{i}\left(1-\bar{\Pi}_{i}\right)}}{\sum_{j=1}^{n} \Delta T_{j} \cdot d_{j}\left(\mathbb{E}\left[N_{T}\right] \bar{\Pi}_{j}+\rho_{j} \sqrt{\operatorname{var}\left(N_{T}\right) \bar{\Pi}_{j}\left(1-\bar{\Pi}_{j}\right)}\right)}
\end{aligned}
$$

The term $\operatorname{var}\left(N_{T}\right)$ in (22) is usually much smaller than $\mathbb{E}\left[N_{T}\right]$, as the underlying names usually have credit qualities above the investment grade and the difference between the investment grade level and the best credit level is small. For example, in our numerical experiments, $\sqrt{\operatorname{var}\left(N_{T}\right)} / \mathbb{E}\left[N_{T}\right] \approx 4 \%$. Because of the good credit qualities, $\bar{\Pi}_{i}>0.5$. Therefore, $\sqrt{\bar{\Pi}_{i}\left(1-\bar{\Pi}_{i}\right)}<\bar{\Pi}_{i}$. To obtain an intuitive feeling for the size of $\partial s / \partial \rho_{i}$, we omit the relatively small terms in the denominator and the minor effects of $\bar{\Pi}_{i}$ and $\Delta T_{i} \cdot d_{i}$, and approximate (22) by

$$
\frac{\partial s}{\partial \rho_{i}} \approx-s \frac{\Delta T_{i} \cdot d_{i} \sqrt{\operatorname{var}\left(N_{T}\right)} \sqrt{\bar{\Pi}_{i}\left(1-\bar{\Pi}_{i}\right)}}{\sum_{j=1}^{n} \Delta T_{j} \cdot d_{j} \mathbb{E}\left[N_{T}\right] \bar{\Pi}_{j}} \approx-s \frac{\sqrt{\operatorname{var}\left(N_{T}\right)}}{n \mathbb{E}\left[N_{T}\right]}
$$




\begin{tabular}{|c|ccc|}
\hline Pool & $\rho_{i}$ & Spreads (bps) & Rel Err (\%) \\
\hline \multirow{3}{*}{ Homogeneous } & -1 & {$[107.37,36.31,15.03,6.40]$} & {$[2.26,1.14,0.63,0.35]$} \\
& 0 & {$[105.36,35.97,14.96,6.38]$} & {$[0.34,0.21,0.11,0.05]$} \\
& 1 & {$[103.42,35.65,14.88,6.36]$} & {$[1.50,0.70,0.41,0.25]$} \\
\hline \multirow{3}{*}{ Inhomogeneous } & -1 & {$[112.03,37.93,15.43,6.49]$} & {$[2.56,1.26,0.70,0.39]$} \\
& 0 & {$[109.66,37.53,15.34,6.47]$} & {$[0.36,0.21,0.11,0.05]$} \\
& 1 & {$[107.39,37.15,15.25,6.45]$} & {$[1.72,0.81,0.47,0.29]$} \\
\hline
\end{tabular}

Table 5: Sensitivity result

Therefore, the relative error in the spread due to the error in $\rho_{i}$ is

$$
\frac{|\Delta s|}{s} \approx \frac{\left|\Delta \rho_{i}\right| \sqrt{\operatorname{var}\left(N_{T}\right)}}{n \mathbb{E}\left[N_{T}\right]} \leq \frac{2 \sqrt{\operatorname{var}\left(N_{T}\right)}}{n \mathbb{E}\left[N_{T}\right]}
$$

as $\left|\Delta \rho_{i}\right| \leq 2$. Furthermore, the relative error due to the errors in all $\rho_{i}$ is bounded by

$$
\sum_{i=1}^{n} \frac{2 \sqrt{\operatorname{var}\left(N_{T}\right)}}{n \mathbb{E}\left[N_{T}\right]}=\frac{2 \sqrt{\operatorname{var}\left(N_{T}\right)}}{\mathbb{E}\left[N_{T}\right]}
$$

To further illustrate the weak dependence of the spread, $s$, on the correlation coefficients, $\rho_{i}$, we compute the spreads with all $\rho_{i}$ set to $-1,0$ or 1 , respectively. The results are listed

in Table 5, where the values inside each parenthesis correspond to the spreads and relative errors for $m=1,2,3,4$, respectively, for the homogeneous and inhomogeneous $m$ th-to-default FBDS considered above. From the table, we see that the maximum relative error for both FBDS is smaller than $3 \%$, which is less than the bound given by (23). Moreover, note that taking all the $\rho_{i}$ to be 0 gives a fairly good rough approximation to the spread for these two examples.

\section{Conclusion}

In this paper, we introduce a fast hybrid approximation method for FBDS. The terminal default probabilities for the FBDS are converted to the form of the terminal default probabilities for BDS. Based on the conditional independence framework, computing the conditional terminal default probabilities for FBDS are similarly converted to computing the equivalent 
terminal default probabilities for BDS. After we obtain the terminal default probabilities, the

default leg is straightforward to compute. For the premium leg, we combine a Monte Carlo simulation for the correlation coefficients $\rho_{i}$ with an analytic method. The transformation method avoids the large combinatorial problem associated with existing analytic methods. Our approach is a generic method applicable to any model based on the conditional independence framework. The method is particularly effective for FBDS because the spread is not very sensitive on the values of the $\rho_{i}$. The numerical results for both homogeneous and inhomogeneous FBDS show that our method is more effective than both Monte Carlo simulation and existing analytic methods.

We believe that this hybrid approach is applicable to the valuation of other financial instruments. Moreover, we avoid the large combinatorial problem by applying a transformation to the terminal default probabilities in FBDS. This technique can also be applied to other products with similar properties.

\section{References}

[1] L. Andersen. Portfolio losses in factor models: Term structures and intertemporal loss dependence. Working paper, available at www.defaultrisk.com, September 2006.

[2] L. Andersen and J. Sidenius. Extensions to the Gaussian copula: Random recovery and random factor loadings. Journal of Credit Risk, 1(1):29-70, 2004.

[3] L. Andersen, J. Sidenius, and S. Basu. All your hedges in one basket. Risk, 16(11):67-72, 2003.

[4] P. Baheti, R. Mashal, and M. Naldi. Step it up or start it forward. The Journal of Fixed Income, 16(2):33-38, 2006.

[5] J. Hull and A. White. Valuation of a CDO and an $n^{\text {th }}$ to default CDS without Monte Carlo simulation. Journal of Derivatives, 12(2):8-23, 2004. 
[6] I. Iscoe and A. Kreinin. Recursive valuation of basket default swaps. Journal of Computational Finance, 9(3):95-116, 2006.

[7] K. Jackson, A. Kreinin, and X. Ma. Loss distribution evaluation for synthetic CDOs. Working paper, available at www.defaultrisk.com, December 2005.

[8] K. Jackson and W. Zhang. Valuation of forward-starting CDOs. Working paper, available at www.defaultrisk.com, February 2007.

[9] D. Lando. Credit Risk Modeling: Theory and Applications. Princeton University Press, 2004.

[10] J. Laurent and J. Gregory. Basket default swaps, CDOs and factor copulas. Journal of Risk, 7(4):103-122, 2005.

[11] D. Li. On default correlation: A copula approach. Journal of Fixed Income, 9(4):43-54, 2000.

[12] O. Vasicek. Probability of loss distribution. Technical report, KMV Corporation, 1987.

[13] W. Zhang. Valuation of forward-starting basket default swaps. Research paper, available at www.cs.toronto.edu/NA/reports.html, 2007. 